\title{
POLÍTICAS \\ MIGRATORIAS DESPUÉS DEL 11 DE SEPTIEMBRE: LOS CASOS DEL TLCAN Y LA UE
}

\author{
FRANCISCO ALBA Y PAULA LEITE
}

a relación entre los procesos de integración económica y los posicionamientos, sobre los flujos migratorios, es un tema que tomó interés a raíz del establecimiento de negociaciones migratorias entre México y Estados Unidos en 2001. El hecho parecía dar lugar a un acercamiento entre las reglas que rigen el funcionamiento del espacio económico de los países signatarios del Tratado de Libre Comercio de América del Norte (TLCAN) y las que configuran el espacio de la Unión Europea (UE). En México está muy extendida la opinión que considera a la política migratoria de la UE - libre movilidad de los ciudadanos - como la idonea, contemplando el reconocimiento de los flujos mexicanos, por lo que desplazarse en esa dirección es visto como algo deseable; un paso adicional para «completar» el modelo de integración.

Antes de que se diera ese paso, cuya posibilidad y alcance son materia de especulación, los atentados terroristas del 11 de septiembre cancelaron las grandes expectativas que se habían creado al respecto. Este suceso, sin embargo, no cancela el interés por indagar, en una perspectiva comparativa, sobre el manejo de la cuestión migratoria en los bloques económicos del TLCAN y la UE; sólo que ahora en un contexto diferente, marcado por consideraciones de seguridad nacional y lucha antiterrorista.

Inmediatamente después del 11 de septiembre, una de las cuestiones que surgen en diversos ámbitos regionales se refiere a si, como reacción a esos acontecimientos, se observaría un retraimiento de la tendencia hacia fronteras más abiertas — tendencia generalmente atribuida a la creciente integración e interdependencia económicas-. Para algunos observadores, las fronteras regresarían a su función tradicional de «barrera», específicamente frente a los flujos migratorios. Sin embargo, para otros, los acontecimientos del 11 de septiembre podrían recomponer, selectivamente, los procesos regionales de integración. El hecho ineludible es que, en el horizonte posterior al 11 de septiembre, las políticas migratorias, comerciales y otras tantas están siendo reevaluadas a través de la lente de la seguridad nacional. 
El propósito de este ensayo es contribuir a una discusión informada sobre los alcances y limitaciones de las enseñanzas que pueden ser extraídas de distintos diseños institucionales muy diferentes. Muchas de las opiniones, que sugieren a la UE como ejemplo orientador para una evolución deseable de América del Norte, parecen desconocer que los proyectos de integración de estos dos bloques parten de filosofías, políticas, objetivos y metas muy diferentes.

Por lo anterior, para mostrar las complejidades, similitudes y diferencias, se juzga importante hacer un análisis comparativo de las situaciones y políticas en ambos bloques con anterioridad al 11 de septiembre de 2001. De esa breve revisión se derivan algunas paradojas, como es el hecho de la porosidad real de las fronteras en el espacio del TLCAN, aunque esa realidad no cuente con un reconocimiento formal.

El terrorismo indudablemente incrementó la conciencia y los miedos -en particular entre el público estadounidense- sobre la porosidad de las fronteras. En la búsqueda de indicios sobre posibles desarrollos futuros en América del Norte, resulta de interés, por lo tanto, examinar también la experiencia de la UE al respecto, por lo menos como un punto de referencia comparativo.

El ensayo revisa, en primer término y de manera secuencial, la experiencia de la UE antes y después del 11 de septiembre, ya que, como se mostrará, si bien se observan cambios, las políticas migratorias no representan cambios cualitativos. La experiencia del espacio del TLCAN se presenta en dos incisos diferentes: en el primero de ellos se examina la concepción prevaleciente sobre la migración en la constitución del espacio del TLCAN y se le da cabida al episodio de las negociaciones migratorias bilaterales entre México y Estados Unidos; en el segundo se examinan las iniciativas y acciones que se han emprendido con posterioridad al 11 de septiembre. Se concluye con una discusión sobre posibles escenarios e implicaciones de lo anterior, y con un breve comentario sobre la transferibilidad de las experiencias.

\section{LA EXPERIENCIA EUROPEA}

\section{ANTECEDENTES GENERALES}

Desde su fundación, en 1957, la Unión Europea se define como un espacio donde se ejercen cuatro libertades fundamentales: libre circulación de bienes, personas, capitales y servicios. ${ }^{1}$ En particular, bajo la libre circulación de personas subyace la de trabajadores, pues se pretende crear condiciones para una utilización óptima de los recursos laborales dentro del espacio comunitario. Esta política establece una clara diferenciación entre la movilidad de ciudadanos comunitarios y no comunitarios; por ende, se establece una diferenciación entre las políticas que regulan la movilidad interna y las que regulan la inmigración de países extracomunitarios.

El proceso de integración comunitaria, sin embargo, se ha desarrollado lenta y progresivamente, lo que se ha reflejado en la evolución del fenómeno migratorio y en el tipo de políticas para su manejo. Hasta 1973, los países que entonces constituían la

${ }^{1}$ A lo largo del ensayo se usará el término Unión Europea (denominación actual), si bien a veces se conservará el de Comunidad Europea o alguna otra de sus denominaciones anteriores. 
Comunidad Europea, en franca expansión económica, implementan políticas activas de reclutamiento laboral de carácter temporal. Los países mediterráneos menos desarrollados (Portugal, España, Italia — miembros de la Comunidad—, Yugoslavia, Turquía, Marruecos, Argelia, Túnez) constituyen los principales lugares de origen de los migrantes, en virtud de las sustanciales diferencias socioeconómicas —industrialización, empleo y salarios- en comparación con los países comunitarios de destino.

La crisis del petróleo de 1973 produce una profunda recesión económica a nivel mundial e induce, a los países reclutadores de mano de obra de Europa occidental, a implementar políticas migratorias restrictivas e, incluso, a incentivar el regreso de los inmigrantes a sus países de origen. Sin embargo, en la práctica, dichas acciones no redundan en una disminución significativa del volumen de inmigración, ya que, por una parte, el retorno de los migrantes «temporales» es difícil de inducir y, por otro lado, porque emergen nuevos canales de inmigración, como son los procesos de reunificación familiar, asilo e inmigración ilegal.

Un protocolo anexo al Tratado de Ámsterdam, en vigor desde el 1 de mayo de 1999, que permitió la integración del Acervo de Schengen a la Unión Europea, cristalizó el objetivo de libre circulación de personas prácticamente en todo el espacio comunitario. ${ }^{2}$ Hasta entonces, el manejo del fenómeno migratorio era materia de competencia nacional. La abolición de fronteras, vigente desde 1995, en los países signatarios de los Acuerdos de Schengen, resultante de la implementación del Mercado Único, operacionalizó en el espacio comunitario la diferenciación entre los movimientos que involucran a los ciudadanos comunitarios y a los ciudadanos de terceros países al desarrollarse políticas regulatorias de los dos tipos de movilidad: interna y externa. Con anterioridad, se había iniciado una cooperación técnica y política entre los países signatarios de la Convención de Schengen, que funcionó como un «laboratorio» del Mercado Único para la libre circulación de personas, a través de la abolición de las fronteras interiores y de la creación de una frontera externa común. ${ }^{3}$

Es relevante señalar que la liberalización del movimiento de personas ocurre en un momento en que las diferencias económicas, entre los países comunitarios —si bien persisten-, ya no son lo suficientemente profundas como para producir una migración a gran escala de los Estados miembros menos desarrollados hacia los más desarrollados. A ello se agrega el elevado costo de salir del país de origen y la existencia de fundadas expectativas de mejoría de las condiciones de vida a futuro, lo que desincentiva el impulso a emigrar (Tassinopoulos y Werner, 1998; Alba, 1998; Fertig y Schmidt, 2002).

La convergencia económica y social entre los países fue considerada, desde un principio, condición necesaria para una integración económica exitosa. Por esa razón, la Comunidad ha participado, mediante un sistema de apoyos «estructurales»y soli-

${ }^{2}$ La especificidad del Reino Unido e Irlanda - que no han firmado los Acuerdos de Schengen- es reconocida en el Tratado, por lo que estos países están autorizados a mantener el control de sus fronteras.

${ }^{3}$ El «espacio Schengen», ratificado en 1985 por Alemania, Francia, Bélgica, Luxemburgo y Holanda -originalmente un acuerdo de ámbito intergubernamental establecido fuera del marco comunitario-, pasa a abarcar, gradualmente, casi todos los Estados miembros de la Comunidad: Italia se adhiere el 27 de noviembre de 1990; Portugal y España, el 25 de junio de 1991; Grecia, el 6 de noviembre de 1992; Austria, el 28 de abril de 1995, y Suecia, Finlandia y Dinamarca, el 19 de diciembre de 1996. Si bien este último país no ha ratificado todavía el Acuerdo. 
darios, en los esfuerzos de desarrollo nacionales para que el conjunto de las regiones y ciudadanos se beneficie de una prosperidad compartida. Como resultado de las políticas de convergencia, los países comunitarios mediterráneos experimentan una reducción de la emigración y emergen, también ellos mismos, como destinos atractivos para migrantes de terceros países.

Antes de proceder a un análisis del proceso de construcción de la política migratoria comunitaria es conveniente señalar que el sistema político de la Unión se encuentra en fase de desarrollo. La UE es un espacio político «compromisorio» y no un espacio deliberativo, es decir, es un espacio donde se estipulan orientaciones comunes que comprometen a los Estados miembros a transponerlas a sus sistemas políticos nacionales, correspondiendo a éstos definir sus propias políticas nacionales, en armonía con las políticas comunitarias.

\section{HACIA UNA POLÍTICA COMUNITARIA \\ DE INMIGRACIÓN Y ASILO}

Si hasta inicios de los años noventa la inmigración extracomunitaria en la UE es una cuestión tratada a nivel de competencia nacional, la abolición de fronteras entre los Estados miembros y la creación de una frontera externa común, en virtud de los Acuerdos de Schengen, coloca a la inmigración como un problema compartido, por lo que se empieza a discutir, más sistemáticamente, la necesidad de implementar una política común de inmigración y asilo.

Los Estados signatarios, bajo una visión pragmática y funcionalista, acuerdan establecer una cooperación técnica y política donde se articulan medidas de control externo e interno. Dentro de las medidas estipuladas para el control externo destacan: la definición común de las condiciones de cruce de las fronteras externas; la separación, en los aeropuertos y puertos, entre los viajeros del interior, del «espacio Schengen», y los provenientes de territorios fuera de ese espacio; la obligación de declarar para aquellos quienes pertenezcan a terceros países que circulan de un país a otro; la definición de normas relativas a la responsabilidad de solicitudes de asilo; la institución de una coordinación entre las administraciones para fiscalizar las fronteras; el refuerzo de la cooperación judicial y la creación del Sistema de Información de Schengen (SIS). ${ }^{4}$

El desarrollo de medidas de control interno se deriva de las deficiencias e imperfecciones del sistema de control externo. Estas medidas pretenden detectar y regular a los que entraron ilegalmente a un país y a los que ahí permanecieron en condición ilegal. Son medidas encaminadas a controlar el mercado laboral, sancionar a los empleadores de inmigrantes en situación irregular y restringir el acceso de los inmigrantes ilegales a los sistemas de bienestar. Una modalidad de control interno de índole distinta se refiere a los procesos de regularización de inmigrantes en situación ilegal (Brochmann, 1999).

Para algunos autores esta lógica funcionalista mostró debilidades debido a limitaciones de dos tipos: por un lado, institucionales — vinculadas con la toma de

${ }^{4}$ Sistema central de información, alimentado por conducto de redes nacionales con vista a garantizar el intercambio de datos relativos a personas y objetos buscados. 
decisiones por unanimidad, propia de la naturaleza de la cooperación intergubernamental- y, por otro, políticas - relacionadas con cierta debilidad de la legitimidad democrática y con la estrechez de la visión estratégica (Pastore, 2002). El empeño en reducir la inmigración legal y en reforzar las estrategias de control tuvo el efecto perverso de dar lugar a contraestrategias, cada vez más sofisticadas y camufladas, para sortear las dificultades impuestas. En los últimos años la inmigración indocumentada ha representado una importante modalidad de inmigración. Se estima que la UE recibe, anualmente, casi tantos inmigrantes legales como indocumentados: alrededor de 500 mil por cada grupo.

El Tratado de Ámsterdam pretende ser una respuesta favorable de los Estados miembros a las presiones de la opinión pública y otras fuerzas políticas. Así, se pretende lograr una mayor seguridad y estabilidad dentro del espacio comunitario. El Tratado establece el objetivo de mantener y desarrollar un área de libertad, seguridad y justicia con la pretensión de asegurar la libre movilidad de los ciudadanos comunitarios, conjugando medidas con respecto a los controles de la frontera externa, el asilo, la inmigración, la prevención y el combate del crimen transfronterizo.

El concepto de un área de libertad, seguridad y justicia implica una clara distinción entre seguridad interior y el entorno exterior, constituyendo las fronteras comunitarias, la línea divisoria que frena la entrada de terceros en la «fortaleza europea». La introducción del concepto de seguridad interior obliga a la «comunitarización» de aspectos que se inscriben en el marco de la Justicia y los Asuntos del Interior, ${ }^{5}$ ámbito en el cual se incluye la cuestión migratoria y el asilo. ${ }^{6}$ El Tratado de Ámsterdam, al incorporar el Acervo de Schengen en el marco institucional y jurídico de la Unión, lo hace objeto de control parlamentario y judicial.

Así, el Tratado de Ámsterdam formaliza, al nivel comunitario y bajo el signo de seguridad interna, una dinámica de inclusión vs. exclusión de ciudadanos, lo cual tiene profundas implicaciones para los ciudadanos de terceros países que desean residir y trabajar en la Unión (Monar, 2000). Esta tendencia incluyente-excluyente de la UE es, de algún modo, comprensible y característica del proceso de integración comunitaria. Cualquier Estado-nación procede a la distinción entre sus ciudadanos y los extranjeros, y, a partir de ahí, garantizar la seguridad de sus ciudadanos es una de sus prioridades. En la medida que se delegan más funciones y competencias en la UE, es de esperar que le competan más responsabilidades en el dominio de la seguridad interna.?

\footnotetext{
${ }^{5} \mathrm{La}$ «comunitarización» corresponde a la transferencia de un sector que depende, en el marco institucional de la Unión Europea, del método intergubernamental al método comunitario. El Tratado de Maastricht creó un pilar referente al campo de la Justicia y Asuntos Internos. Al mostrarse este pilar, claramente inefectivo en la regulación de la inmigración y el asilo, el Tratado de Ámsterdam busca superar esa ineficacia, consagrando la competencia comunitaria en esas materias. El desarrollo, en el marco comunitario de políticas en el ámbito de la Justicia y Asuntos del Interior, es considerado una innovación del Tratado de Ámsterdam y un avance en el proceso de integración política de la Unión.

${ }^{6}$ El Tratado de Ámsterdam introduce, en el Tratado Constitutivo de la Comunidad Europea, el título IV (artículos 61 al 63), denominado «Visados, asilo, inmigración y otras políticas relacionadas con la libre circulación de personas», que comprende los siguientes ámbitos: libre circulación de personas; controles de fronteras exteriores; asilo, inmigración y protección de los derechos de los nacionales de terceros países; cooperación judicial en materia civil.

${ }^{7}$ Dada la renuencia de los Estados miembros a ceder soberanía en materia de su seguridad interna, el
} 
Las directrices, para la implementación de una Política Común de Inmigración y Asilo, fueron desarrolladas en el Consejo Europeo de Tampere (15 y 16 de octubre de 1999), dedicado a debatir la creación de un espacio de libertad, seguridad y justicia en la UE. Frente a las críticas sobre la «fortaleza Europea», el Consejo de Tampere ofreció un posicionamiento más liberal, al afirmar el objetivo de una Unión Europea «abierta y segura». La flexibilización del posicionamiento comunitario se desprende del reconocimiento de que el modelo de «inmigración cero», implementado desde los años setenta, es irrealista y desfavorable, dado que, por un lado, ha «incentivado» la inmigración irregular y la utilización abusiva de los sistemas nacionales de asilo y, por otro lado, es ineludible la necesidad de inmigrantes extracomunitarios.

Sin embargo, la nueva matriz de la política migratoria recalca que la integración de los inmigrantes tendrá que ser acompañada por un refuerzo de la frontera externa de la Unión a fin de garantizar una inmigración selectiva, factible de ser integrada económica, social y culturalmente.

Los lineamientos de la Política Común de Migración y Asilo del Consejo de Tampere muestran un abordaje comprensivo del fenómeno migratorio al incluir los ámbitos de la gestión de los flujos inmigratorios, el sistema de asilo, la integración de los inmigrantes y la cooperación con los países de origen y tránsito. La tónica no es la de detener la inmigración, sino manejarla a fin de maximizar sus beneficios y minimizar sus eventuales efectos adversos. Lo anterior presupone privilegiar los canales legales de inmigración laboral, considerando las reales necesidades del mercado de trabajo, el combate a la inmigración indocumentada, la aproximación del estatuto de los ciudadanos de terceros países al de los nacionales y la cooperación con los países de origen y tránsito.

La colaboración con los terceros países se ajusta a una lógica que busca prevenir (y excluir del espacio comunitario) la migración en el origen, a través del establecimiento de acuerdos bilaterales y multilaterales que posibiliten la «externalización» del control migratorio, además de estrategias de codesarrollo con el propósito de reducir las causas de la migración. En la práctica, los progresos en todos los ámbitos contemplados han sido reducidos y la implementación de la Política Común de Inmigración y Asilo se encuentra en un lento proceso de desarrollo.

Una primera explicación de los limitados progresos se relaciona con la dicotomía soberanía nacional $v s$. soberanía supranacional. La soberanía nacional sigue desempeñando un papel crucial en la definición de la política migratoria y de los mecanismos de regulación del fenómeno. ${ }^{8}$ Otras explicaciones se refieren a que existen sustanciales dis-

marco comunitario de justicia y asuntos internos se encuentra bajo un «régimen especial» que combina las competencias nacional, intergubernamental y supranacional, lo que resulta en una mayor complejidad y desafío para la coordinación y concreción de políticas comunes (Monar, 2000, «Justice and Home Affairs in a wider Europe: the dynamics of inclusion and exclusion» en ESRC One Europe or Several? Programme Working Paper 07/00, Sussex European Institute; Spencer, 2001, «The EU as a security actor in the Mediterranean: problems and prospects» en Fred Tanner (ed.), The EU as a security actor in the Mediterranean, ETH, Zurich, pp. 9-30; Shaw, 2002, «Sovereignty at the boundaries of the politic», ESRC One Europe or Several? Programme Working Paper 44/02, Sussex European Institute).

${ }^{8}$ El Consejo Europeo tiene la competencia exclusiva para legislar a partir de las propuestas de la Comisión Europea y las decisiones deben ser tomadas por unanimidad. Obviamente, esto dificulta de manera enorme la definición de una política común, ya que cualquier Estado puede ejercer su derecho de veto. 
crepancias en la materia entre los Estados miembros, lo que es entendible si se consideran los contextos - económico, social, cultural — en que ocurre la migración; algunos países tienen una larga experiencia migratoria, mientras que en otros el fenómeno es reciente.

Ciertos países, por su ubicación geográfica (por ejemplo, los países mediterráneos), son más vulnerables a la inmigración ilegal. La estructura del mercado laboral en algunos países demanda trabajadores altamente calificados, mientras que en otros subsiste una fuerte demanda por trabajadores poco calificados e, incluso, ilegales, dado el peso de la economía subterránea. Con relación al asilo, existen también entre los Estados miembros diferencias profundas, lo que inhibe la adopción de una política de admisión común (Pastore, 2002).

Los cambios políticos, que han ocurrido en ciertos países después de Tampere, hacen que el «consenso» político, en el interior de cada país y entre los Estados miembros, se haya estructurado en torno a políticas defensivas y de carácter restrictivo. A ello han contribuido las respuestas a los acontecimientos del 11 de septiembre.

\section{DespuéS DEL 11 DE SEPTIEMBRE}

El desarrollo de una política integral —y más liberal, de inmigración y asilo- ha sido obstaculizado por los atentados terroristas del 11 de septiembre al ganar terreno las perspectivas de orden público y de seguridad interna. La «securización» del fenómeno migratorio y del asilo encuentra, así, su legitimidad reforzada.

En el Consejo Extraordinario de Justicia y Asuntos Internos, celebrado el 20 de septiembre de 2001, se propone que la Comisión «examine urgentemente la relación entre la defensa de la seguridad interna y las obligaciones de protección internacional y sus instrumentos» (JHA Council, 2001, conclusión 29). En respuesta, la Comisión presenta un documento donde invita a los Estados miembros a aplicar rigurosamente las cláusulas de exclusión previstas por la Convención de Refugiados y les señala, al mismo tiempo, instrumentos legales que les permiten recusar la admisión de nacionales de países terceros por motivo de política pública o seguridad interna, incluyendo los casos de inmigración legal, reunificación familiar, residencia de larga duración y visas para estudiantes (COM, 2001).

El Consejo de Sevilla, celebrado el 21 y 22 de junio de 2002, centra su atención en el aspecto más mediatizado y politizado del fenómeno migratorio: la inmigración indocumentada. De este modo, el Consejo establece, como primera prioridad, la lucha contra la inmigración ilegal al enfocarse en tres aspectos: la repatriación de inmigrantes ilegales, la gestión coordinada e integrada de las fronteras exteriores y la lucha contra el tráfico de personas. Bajo la presidencia española, los países comunitarios del sur y el Reino Unido adoptan un dispositivo conjunto de control marítimo de fronteras para combatir el tráfico ilegal de inmigrantes en el Mediterráneo, mismo que se pretende extender al Atlántico, entre las costas subsaharianas y las Canarias.

Siguiendo la directriz comunitaria de invocar la colaboración de los terceros países, se pretende que los Estados miembros establezcan acuerdos bilaterales con los principales países de origen, con el objetivo de manejar los flujos desde su fuente. En teoría los acuerdos aspiran a un abordaje integral de las diferentes dimensiones del fenómeno migratorio - entrada de trabajadores, reunificación familiar, retorno, control de fronteras, repatriación y codesarrollo-. En la práctica esos acuerdos se restringen a 
un reclutamiento legal muy limitado de trabajadores, a la cooperación en los controles fronterizos para frenar la migración ilegal y al establecimiento de acuerdos de readmisión de inmigrantes indocumentados y de asilados.

El 11 de septiembre ha sustanciado la securización de los movimientos migratorios, al mismo tiempo que ha reforzado la lógica que atribuye a los inmigrantes y asilados un papel de potenciales amenazas a la seguridad del Estado y la sociedad. El resurgimiento de la soberanía nacional como factor crucial en el ámbito político, en virtud del clima de inseguridad generado a partir del 11 de septiembre, conduce nuevamente a un enfoque más restrictivo de los movimientos migratorios y, por ende, a una mayor dificultad de convergencia política entre los Estados miembros.

Sin embargo, en la conferencia intitulada Manejo de la migración en beneficio de Europa, realizada el 15 y 16 de mayo de 2003, bajo los auspicios de la presidencia griega, se plantea que la inmigración se inscribe en el futuro de Europa y que es forzoso abandonar las políticas altamente restrictivas para optar por políticas selectivas e inclusivas. Se considera que el debate sobre migración en Europa no puede ser monopolizado por el combate a la inmigración ilegal, lo que tiene el efecto de exacerbar viejos miedos y alimentar sentimientos xenófobos y racistas en la opinión pública. En esa conferencia, el ministro griego de Asuntos Exteriores sostuvo que: «No solamente necesitamos fronteras seguras, necesitamos fronteras inteligentes [...] Necesitamos fronteras que reciban a los inmigrantes que nuestras sociedades requieren, así como a los refugiados que nuestros deberes legales e instintos humanitarios nos obliguen a proteger» (AMPI News, 2003).

A tres años de distancia de los ataques terroristas del 11 de septiembre en Estados Unidos, la apertura del debate, sobre el manejo más adecuado de la inmigración y el asilo, parece ser un desarrollo favorable hacia una política más liberal e integral, tal como fue definida en Tampere.

\section{LA SITUACIÓN PREVIA AL 11 DE SEPTIEMBRE:}

\section{DEL TLCAN A LAS NEGOCIACIONES MIGRATORIAS}

De forma muy diferente a los tratados que han ido paso a paso profundizando la integración europea, el TLCAN no busca sino crear una zona de libre comercio; no es el primer paso de un proyecto más ambicioso, comparable al europeo. El paradigma que subyace tras la implementación del TLCAN es el de un «manejo corporativo», en el que se acepta un elevado nivel de integración económica bajo condiciones de separación política y sin instituciones de «gobierno colectivo». Sin embargo, aún no entraba en vigor el TLCAN, cuando ya se especulaba sobre posibles implicaciones en otros ámbitos no contemplados en el mismo. En particular, interesaron a muchos observadores las implicaciones que pudieran derivarse en términos de tendencias y políticas frente a los flujos migratorios desde México. De hecho, el TLCAN fue «vendido» al Congreso estadounidense, en parte, porque se le convenció que con la liberalización comercial estos flujos disminuirían de intensidad y se volverían más manejables. La cuestión migratoria y la frontera eran puntos difíciles de la relación entre Estados Unidos y México. ${ }^{9}$

${ }^{9}$ Este escenario fue puesto en duda por múltiples analistas, entre otros, por Alba (1993) y García y Griego (1993). 
La frontera entre Estados Unidos y Canadá tradicionalmente no se había visto como problemática; se caracterizaba por reglamentaciones sobre inmigración y turismo comparativamente muy «laxas» (permissive). Como afirma un analista estadounidense y canadiense, observador participante: «antes del 11 de septiembre, todos cruzábamos la frontera de manera rutinaria. Ocasionalmente, funcionarios de aduanas e inmigración, de ambos lados, nos recordaban que esa frontera era una frontera internacional seria, sobre todo cuando viajábamos con un visitante cuya ciudadanía no era ni canadiense ni estadounidense» (Pauly, 2003).

La entrada en vigor del TLCAN ejerció una enorme presión sobre la frontera entre Estados Unidos y Canadá, y obligó a una «primera ronda» de reflexiones sobre su función y significado en un contexto mayormente integrado e interdependiente. La mayoría de las poblaciones locales y los gobiernos consideraron aceptable el emergente statu quo - de una creciente movilidad transfronteriza de bienes, capital y determinadas categorías poblacionales-. Más aún, para darle acomodo a estas realidades, Ottawa comenzó a urgir a Washington a tomar medidas conjuntas para mejorar la seguridad de la frontera (detectando migrantes peligrosos) e incrementar la eficiencia (acelerando el paso de cargamentos confiables y concentrando el personal en los envíos de mayor riesgo). Conversaciones sobre un «partenariado fronterizo Canada-Estados Unidos» habían sido iniciadas con la administración de Clinton, pero progresaron poco (Clarkson, 2003).

La experiencia en la frontera entre Estados Unidos y México fue muy diferente. Muy pocos imaginaron el desarrollo «dicotómico» que estaba por ocurrir, un desarrollo lleno de paradojas. Desde la perspectiva de Estados Unidos, se requería una frontera eficiente para el tráfico comercial, pero infranqueable para todo aquello no formalmente autorizado a hacerlo. Este doble propósito se encapsuló en la estrategia de facilitation and control. Sin embargo, esta estrategia encerraba una gran contradicción pues se pretendía, por un lado, el control de flujos migratorios que, por otra parte, se incentivaban por las implicaciones de la liberalización comercial que se impulsaba agresivamente (Massey, 1998; Massey et al., 2002). De hecho, reflejo de ese contexto, en la frontera México-Estados Unidos se manejó, de manera satisfactoria, una triplicación de los flujos comerciales de alrededor de 80 mil millones, en 1993, a cerca de 250 mil millones en 2000, la mayor parte de los cuales cruzan la frontera en vehículos de todo tipo. Desde el comienzo del TLCAN se han abierto diez nuevos cruces fronterizos. Pero esta frontera amigable y muy ajetreada fue la otra cara de una frontera convertida en barrera defensiva, crecientemente vigilada y subrepticiamente militarizada - para el control de la frontera sur de Estados Unidos, la estrategia dominante fue la de «prevención por medio de la disuasión».

Aún no entraba en vigor el TLCAN cuando, en 1993, se ensaya la Operation Blockade, más adelante renombrada como Operation Hold the Line, en la zona de El Paso, Texas. A partir de esa medida se incrementa la dotación de recursos a la Patrulla Fronteriza y se continúa con una serie de «operativos» que bloquean, con bardas múltiples y sofisticados equipos de vigilancia, los puntos de cruce más concurridos por los indocumentados entre el Océano Pacífico y el Golfo de México.

Para no impedir la continuación de la integración económica (de la producción) a lo largo de la frontera, también se ponen en operación las líneas «SENTRI» (sistema electrónico seguro para una inspección rápida de viajeros) en Otay Mesa, California. Este sistema utiliza medios tecnológicos avanzados para permitir el cruce, sin verifica- 
ción de documentos, en líneas especiales a viajeros considerados de bajo riesgo (como ejecutivos de las plantas maquiladoras).

Con el tiempo, el TLCAN también alimentó expectativas que hicieron pensar que las problemáticas de la migración y de la frontera México-Estados Unidos pudieran ser superadas. El presidente Vicente Fox, recién electo, articuló una propuesta para una nueva relación especial con Estados Unidos y Canadá, basada en una profundización del TLCAN, inspirada en el modelo de la UE. El objetivo era elevar de nivel las relaciones con Estados Unidos, pasando de asociado comercial a aliado estratégico y vecino confiable, lo que implicaba una estrecha cooperación en la lucha contra el narcotráfico y, también, en el área de seguridad. A cambio, México esperaba una solución bilateral para la cuestión migratoria: regularización para los, aproximadamente, 3.5 a 4 millones de mexicanos indocumentados con residencia en Estados Unidos; algún relajamiento de las medidas draconianas de control a lo largo de la frontera, mismas que colocan a los mexicanos frente a riesgos desmedidos en los cruces no autorizados de la frontera, y la negociación de algún programa o programas de trabajadores temporales.

En febrero de 2001, los presidentes Fox y Bush acuerdan iniciar negociaciones migratorias a fin de llegar a «un esquema ordenado de flujos migratorios». ${ }^{10}$ Las negociaciones migratorias emprendidas venían a subsanar un importante vacío en la relación bilateral. Se trataba de la «normalización» de uno de los nexos que vinculan profundamente a ambos países. Al buscar reglas para su manejo, se le otorgaba al fenómeno migratorio un tratamiento similar al otorgado a los intercambios comerciales, de inversión y financieros, enfoque que en su momento culminó en el TLCAN.

Hay indicios inequívocos de que existió voluntad política por ambas partes de llegar a resultados concretos en un periodo razonable. No obstante, las negociaciones no llegaron a materializarse en acuerdo alguno. Los acontecimientos del 11 de septiembre de 2001 vinieron a abortar cualquier avance que se pudiera haber conseguido. ${ }^{11}$

Sin embargo, si bien las negociaciones migratorias han sido archivadas, sus significados y legados marcan fuertemente la discusión actual sobre la cuestión migratoria. Que el gobierno de Estados Unidos se sentara a la mesa de las negociaciones alentó el reconocimiento, por parte de aquel país, que su economía ejerce una demanda sostenida por trabajadores migrantes mexicanos, cuya contribución económica y social es apreciada («Rethinking Mexican Immigration», editorial, The New York Times, 23 de julio de 2001).

El hecho mismo de que se entablaran las negociaciones permitió que la posición de México se expresara de forma abierta, concreta y precisa. En el pasado, México se había encerrado en el principio universal y abstracto de la defensa de los derechos de los migrantes mexicanos. Las negociaciones obligaron a la parte mexicana a traducir sus principios y objetivos tradicionales - el respeto a los derechos de los migrantes y el mantenimiento de una salida confiable para parte de sus trabajadores - en las propuestas concretas que constituyeron los puntos de la agenda de la negociación.

Por lo que se refiere a las implicaciones y los legados, permanece el reconocimiento por parte no sólo de los gobiernos, sino de múltiples actores políticos de los dos

${ }^{10}$ Hacia una prosperidad compartida. La propuesta de Guanajuato, 16 de febrero, 2001.

${ }^{11}$ El camino para alcanzar algún acuerdo aún era largo. El Congreso de Estados Unidos se encontraba muy dividido sobre los diferentes puntos de la agenda de negociaciones. 
países de la realidad y funcionalidad del fenómeno migratorio ante las fuerzas económicas y demográficas que subyacen a esa realidad. La realización de negociaciones ha tenido el efecto de agente catalizador para la búsqueda de respuestas frente al fenómeno migratorio, tanto en México como en Estados Unidos.

En Estados Unidos sobresale el reclamo al presidente para que cumpla con su promesa de negociar un acuerdo migratorio histórico con México (para satisfacer las necesidades laborales y los intereses económicos y de seguridad de ese país) (The New York Times, mayo 19 de 2003, p. A22).

En México, los llamados a la negociación y la exigencia de un acuerdo migratorio se han vuelto parte de las agendas de todos los partidos políticos, de los medios de comunicación y de casi todos los líderes de opinión. ${ }^{12}$ El gobierno mexicano no puede dejar de insistir ante el estadounidense en la necesidad de negociar un acuerdo migratorio, que supere el actual y altamente costoso statu quo.

\section{SEguridad NACIONAL y FRONTERAS EN AMÉRICA DEL NORTE DESPUÉS DEL 11 DE SEPTIEMBRE}

La respuesta inmediata de Estados Unidos, a los ataques terroristas del 11 de septiembre de 2001, fue cerrar sus fronteras y espacio aéreo. Cuando se volvieron a abrir, lo hicieron bajo un nuevo contexto: crecientes y más estrictos controles fronterizos y un discurso político centrado en la protección de la seguridad nacional. Hasta antes de esos ataques parecía existir un amplio consenso sobre el hecho de que la «globalización» le haría perder sentido a las fronteras. Se daba por sentado que la creciente integración e interdependencia económicas conducirían a fronteras más abiertas. Lo que estaba sucediendo es lo siguiente: desde perspectivas analíticas diversas y de las prioridades de las políticas públicas, los esfuerzos de reconceptualización de las fronteras y su significado estaban tomando un lugar prominente. ${ }^{13}$

La agenda internacional de Estados Unidos cambió, de un día para otro, sus prioridades. La seguridad nacional resurgió como la prioridad número uno en el espacio del TLCAN, porque así lo fue para Estados Unidos. De ahí que los flujos comerciales y migratorios no puedan escapar de ser analizados bajo el lente de la seguridad. De hecho una de las bajas más trascendentes de esos ataques fue la propuesta del presidente Fox de promover, entre los tres socios del TLCAN, un concepto de TLC-plus. México es desbancado del sitio especial que había adquirido en la política exterior del presidente Bush. Las negociaciones se detuvieron y el «momento» político que se había ido construyendo a favor de México, incluyendo un más flexible acomodamiento frente a los flujos migratorios, sufrió un fuerte revés.

Con posterioridad al 11 de septiembre, las fronteras adquieren un significado diferente. Preservar la seguridad nacional y la lucha antiterrorista presentan nuevos retos

\footnotetext{
${ }^{12}$ Las notas en periódicos mexicanos, sobre el tema de un acuerdo migratorio, son «una columna regular» desde que las negociaciones levantaron grandes expectativas de que se podía llegar a uno relativamente amplio, que habría satisfecho en medida significativa las demandas de la agenda mexicana.

${ }^{13}$ La bibliografía al respecto es inmensa y en constante aumento. Una contribución reciente es la del ministro de Asuntos Exteriores de Francia, en la que sostiene que las fronteras, más que barreras, son signos de identidad que ayudan a convivir en la diversidad contemporánea (De Villepin, 2003).
} 
para un reordenamiento y estabilidad de las relaciones internacionales, dadas las profundas y múltiples implicaciones de esta emergente reordenación de prioridades. Desde esta perspectiva, una de las preguntas que se plantean se refiere a las implicaciones, sobre las políticas y los flujos migratorios, de la reconceptualización y reconfiguración de las fronteras, así como de los aparatos burocráticos responsables de su control. ¿Cuál es el nuevo conjunto de retos y limitantes? ¿Cuáles son las nuevas oportunidades?

Por lo que se refiere a los flujos mexicanos y la frontera Estados Unidos-México, éstos volvieron a ser considerados bajo la lente de las prioridades internas de la aplicación de la ley, del orden y de la lucha antiterrorista —ámbitos del Departamento de Seguridad Nacional (Homeland Security), departamento al que estará inscrito el antiguo Servicio de Naturalización e Inmigración (INS, por su siglas en inglés) - . Las partidas presupuestales y el personal para proteger las fronteras se incrementan sustancialmente.

Tal vez, la más elocuente muestra de lo anterior sea el nuevo escrutinio que ha estado recibiendo la frontera Estados Unidos-Canadá, con una triplicación de los agentes asignados a esa frontera y el envío ocasional de la «Guardia Nacional» para ayudar en los patrullajes e inspecciones en los puestos fronterizos. Algunos observadores han llegado, incluso, a caracterizar esta nueva situación en la frontera norte estadounidense como una «mexicanización» de la frontera Estados Unidos-Canadá (Andreas, 2003).

Conformándose al marco de la acción y reacción, descrito para entender las respuestas y políticas sobre las cuestiones migratorias entre México y Estados Unidos (Weintraub et al., 1997), los gobiernos de México y Canadá han respondido a la enorme presión puesta sobre ellos — para asegurar la seguridad estadounidense - con medidas de reforzamiento de sus fronteras, a fin de mantenerlas abiertas para la continuación de los intercambios comerciales que son vitales para las dos economías. ${ }^{14} \mathrm{La}$ reacción de los dos gobiernos ha sido la de colaborar — en un contexto donde Estados Unidos define en gran medida la agenda y en el cual los espacios de maniobra son muy estrechos - a fin de convencer a ese país de que ellos son también parte de la solución frente a la emergente problemática de la seguridad nacional.

Desde el lado canadiense, para demostrar una firme determinación contra el terrorismo y la seguridad fronteriza, el gobierno ha asignado partidas adicionales (por un monto cercano a cinco mil millones de dólares) para el financiamiento de tecnologías de detección, el mejoramiento de la infraestructura fronteriza y el aumento de personal para el fortalecimiento del marco de seguridad (alrededor de dos mil miembros de la policía montada fueron reasignados para el patrullaje de la frontera y trabajo antiterrorista). Además, se introdujo una tarjeta antifraude para los nuevos inmigrantes, se incrementó la capacidad de detención y deportación, se multiplicaron los chequeos para las peticiones de asilo y se restringió el régimen de visas para determinadas nacionalidades (Immigration and Refugee Protection Act, Bill C-11).

Desde el lado mexicano se ha actuado de manera similar, restringiendo la entrada de determinadas nacionalidades y compartiendo información con Estados Unidos sobre potenciales sospechosos. Se está trabajando en la creación de una base de datos

${ }^{14}$ Canadá y México dependen mucho más del comercio con Estados Unidos de lo que depende este país del comercio con sus otros dos socios del TLCAN. Se trata de una interdependencia muy asimétrica. El intercambio comercial anual entre Canadá y Estados Unidos se aproxima a 400 mil millones de dólares; el de México y Estados Unidos, a 300 mil millones. 
sobre migración, modernizando sus sistemas computacionales. También se introducen nuevos detectores de documentos falsos en importantes puntos de cruce de la frontera sur del país. De manera similar a como lo hace Canadá, se trata también de ganarse los favores de Washington, implementando medidas para crear confianza.

Producto de esta actitud de colaboración, Canadá y México, cada uno por su lado, han suscrito con Estados Unidos acuerdos de colaboración fronteriza.

En diciembre de 2001, Canadá y Estados Unidos firmaron el acuerdo Manley-Ridge sobre seguridad fronteriza (The U.S.-Canada Smart Border Declaration), un plan de acción de 30 puntos por el que se comprometen a desarrollar mecanismos bilaterales para mejorar la infraestructura fronteriza, además de poner un énfasis especial en el intercambio de información y en la cooperación como componentes críticos de una frontera común segura. La declaración era muy específica en ciertas áreas y se esperaba que hubiera progresos en su implementación. De hecho, al año de su firma, están en distintas fases de operación tres importantes programas bilaterales: NEXUS, un programa de líneas rápidas para viajeros frecuentes preaprobados; FAST (Free and Secure Trade), un programa de precertificación para realizar, fuera de la frontera, los trámites aduanales y así cruzar en líneas especiales; IBET (Integrated Border Enforcement Teams), un programa destinado a hacer los sistemas de información compatibles, a adoptar estándares comunes, a compartir servicios de inteligencia.

México y Estados Unidos firmaron, por su lado, un acuerdo de 22 puntos sobre seguridad fronteriza en marzo de 2002. Aunque el acuerdo no es «tan avanzado» como el firmado por Canadá y Estados Unidos, representa un paso fundamental en ese acercamiento entre los dos países para trabajar conjuntamente en cuestiones de eficiencia fronteriza. El acuerdo se propone establecer un mecanismo de intercambio de información sobre pasajeros, explorar métodos para facilitar el movimiento de viajeros-TLCAN y sostener conversaciones sobre políticas de visas. Este acuerdo representa para México la obtención de cooperación especial para entrenamiento y transferencia de tecnología para el manejo, eficiente y seguro, de sus fronteras norte y sur - esta última es vista, también, como puerta de entrada al espacio del TLCAN-. Los dos países se han comprometido a expandir las líneas SENTRI y han establecido, asimismo, grupos de trabajo sobre asuntos aduanales y sobre tráfico de extranjeros.

\section{ESCENARIOS E IMPLICACIONES AÚN INDETERMINADOS}

Indudablemente es muy prematuro ver con claridad las implicaciones que tendrá sobre las posturas y políticas migratorias, al interior del espacio del TLCAN, que la migración sea vista, de manera acentuada y en un futuro inmediato, a través de la lente de la seguridad nacional y la lucha antiterrorista. Esta contaminación de temas, que se expresa en la «securización» de la cuestión migratoria, es ya un parámetro ineludible a tomar en cuenta en el manejo y administración de los flujos migratorios.

Con propósitos heurísticos, y desde la perspectiva comparativa de los procesos en los espacios de la UE y el TLCAN, surgen a la consideración dos escenarios extremos en el futuro del espacio del TLC: uno es la emergencia de una «Fortaleza América» —donde América significa Estados Unidos-; otro, el de una «Fortaleza América del Norte» — donde América del Norte se refiere al espacio del TLCAN-. 
La Fortaleza América significa la fortificación unilateral y el endurecimiento de las fronteras de Estados Unidos, donde las consideraciones de seguridad nacional avasallan cualquier otra. Sin embargo, este escenario extremo va en contra de poderosos intereses económicos. De hecho, los gobiernos de Canadá y México, junto con múltiples grupos empresariales de los tres países, se han movilizado para evitar, justamente, la materialización de este escenario.

Por su lado, la Fortaleza América del Norte implica una casi plena armonización trilateral de políticas sobre seguridad, migración, antiterrorismo y áreas vinculadas para crear, formalmente - en una especie de soberanía compartida—, un «Perímetro de América del Norte» que se sobrepondría al espacio del TLCAN. Este escenario equivaldría a una «europeización» de las fronteras exteriores, y presumiblemente interiores, de ese perímetro. Sin embargo, este escenario requeriría tal nivel de institucionalización formal y armonización de políticas, que resulta una opción poco factible en las condiciones políticas actuales de los tres países. Baste mencionar, al respecto, la postura canadiense. Canadá no contempla las cuestiones sobre controles fronterizos, seguridad nacional y flujos migratorios como asuntos trilaterales y prefiere concentrar su atención en el manejo del paralelo 49 (al considerar que las condiciones de las fronteras Canadá-Estados Unidos y México-Estados Unidos son completamente diferentes) (Hristoulas, 2003).

Dados los procesos experimentados a tres años del 11 de septiembre y las posturas de cada uno de los países frente a cuestiones de seguridad nacional y migración, el escenario más factible es uno intermedio entre los dos extremos antes mencionados. La arquitectura de los acuerdos firmados —entre Canadá y Estados Unidos, por un lado, y México y Estados Unidos, por otro- sugiere un proceso de integración adicional —en materia de seguridad - «a dos velocidades»: con mayor profundización y concreción en el caso de Canadá y Estados Unidos; con menos profundidad y mayormente propositivo sobre metas y métodos en el caso de México y Estados Unidos.

Podría resultar paradójico, pero México parece interesado en articular la opción de la trilateralización a fin de asegurar, a la vez, la seguridad en América del Norte y las fronteras abiertas que requiere el espacio económico del TLCAN (Hufbauer y Vega, 2003). La apuesta de México tiene mucha relación con lo que espera obtener a cambio: una liberalización de los flujos migratorios. Sin embargo, la posición de México es también ambigua, ya que se ha tenido una enorme dificultad para acomodar los distintos intereses en juego ante sistemas migratorios de carácter regional que se entrecruzan con los flujos mexicanos. México no es sólo país de origen de migrantes, sino también importante país de tránsito (no sólo de migrantes centroamericanos). En el contexto actual, el país se enfrenta a retos ingentes para no entrar en conflicto con países cercanos culturalmente y de importancia estratégica, pero, asimismo, al país se le ofrecen oportunidades para hacer avanzar sus históricos objetivos en materia migratoria. México tiene que ponderar las contrapartidas de un mayor esfuerzo en el control de sus fronteras.

Por lo que respecta a la postura de Estados Unidos, la interdependencia es tan asimétrica entre él y los otros dos socios del TLCAN —Canadá, por un lado, y México, por otro- que el bilateralismo sigue otorgando ventajas en la definición de agendas y consecución de intereses en beneficio de un Estados Unidos hegemónico, que no tiene interés en institucionalización multinacional alguna que pueda representar una disminución de su plena libertad de maniobra (como se desprende de sus posicionamientos internacionales posteriores al 11 de septiembre, pero también respecto del TLCAN).

Lo que parece irse configurando en el horizonte es el surgimiento de una «Amé- 
rica» protegida por dos Estados «buffer», cada uno con diferentes características dadas sus condiciones particulares, a los que se les pedirá que hagan los respectivos cambios necesarios para garantizar la seguridad nacional de Estados Unidos. Sin embargo, este escenario intermedio refleja un elevado grado de incertidumbre sobre las implicaciones que, finalmente, se deriven para la cuestión migratoria de la integración a la que den lugar las iniciativas sobre seguridad en América del Norte.

La «securización» de la cuestión migratoria fortaleció, en la UE, tendencias comunitarias que ya se venían dando desde la creación del espacio Schengen, si bien las orientó en un sentido más restrictivo frente a terceros. El potencial de influencia de esta securización, en el espacio del TLCAN, es mucho mayor por el papel central que juega Estados Unidos en este espacio, caracterizado por una interdependencia estructuralmente asimétrica. Sin embargo, más allá de esta securización inmediata, las grandes diferencias de los proyectos de integración regional persisten con sus implicaciones, ya establecidas con anterioridad al 11 de septiembre, sobre las políticas migratorias en el espacio de la UE y del TLCAN.

Ello se hace evidente en los planes de ampliación de los espacios respectivos. La ampliación inminente de la UE en 2004, que abarca diez países de Europa Central, Oriental, Báltica y Mediterránea, ${ }^{15}$ constituye un desafío sin precedentes en la historia de la construcción europea. En términos poblacionales, el alargamiento se traducirá en un incremento de cerca de 104 millones de personas, lo que impone inusuales retos a la cohesión de la UE, ya que las diferencias en términos del nivel de desarrollo son mucho más significativas que las observadas en las ampliaciones anteriores. ${ }^{16}$ Dada la magnitud de las discrepancias de ingresos y el elevado esfuerzo de integración económica en juego, existe en el interior de la $\mathrm{UE}^{17}$ gran preocupación de que la libre movilidad de personas resulte en una migración masiva, con un impacto adverso sobre el mercado laboral y los ingresos de los 15 Estados miembros.

Sin embargo, la respuesta de la UE no ha sido diferente de las negociadas en el pasado, desde la fundación de la entonces Comunidad Económica Europea: establecer un periodo transitorio, relativamente corto, antes de que entre en efecto la libre movilidad de los trabajadores y las personas. Durante ese periodo se espera que la mayor integración económica, apoyada por políticas públicas —nacionales y comunitariasde cohesión social, proporcione convergencia económica de los nuevos miembros ha-

\footnotetext{
${ }^{15}$ Eslovenia, República Checa, Eslovaquia, Polonia, Hungría, Estonia, Letonia, Lituania, Malta y Chipre.

${ }^{16}$ Los diferenciales de PIB per capita, a paridad del poder adquisitivo entre los 15 Estados miembros y los 10 países candidatos, son bastante significativos: según estimaciones de Eurostat (2000), los ingresos de los países candidatos representan el $40 \%$ de los de la UE. No obstante, los países candidatos se diferencian sustancialmente entre sí; por ejemplo, el PIB per capita constituye para Bulgaria el 20\%, mientras que para Eslovenia el 60\% de lo que caracteriza la UE. Los salarios en los 10 países son bastante más bajos; varían entre el 20 y el 60\% respecto de los salarios de la UE.

${ }^{17} \mathrm{La}$ UE propone una política de integración prudente, estipulando un periodo transitorio de cinco años, en el cual no entra en vigor la libre circulación de personas. Posteriormente, existe un periodo de dos años para proceder a la evaluación de las condiciones de los mercados laborales de los Estados miembros. En el caso de que un Estado miembro determine que su mercado de trabajo no está preparado para la libre movilidad de personas del este, podrá solicitar el alargamiento del periodo transitorio. Lo anterior significa que la libre circulación de personas para los 10 Estados miembros podrá ser postergada hasta el 2010 y que en el periodo transitorio los 15 podrán establecer cuotas de inmigración para los nuevos países (Boeri y Brücker, 2001; Boeri et al., 2002; OECD, 2002).
} 
cia los niveles comunitarios. En virtud de lo anterior, la liberalización de movimientos de los nuevos ciudadanos comunitarios entrará en vigor en 2010.

El equivalente a la ampliación del espacio del TLCAN es el proyecto de un Acuerdo de Libre Comercio de América (ALCA), una iniciativa de integración hemisférica muy ambiciosa, difícil y compleja. Sin embargo, el ALCA, al igual que el TLCAN, se reduce a establecer una zona de libre comercio; ninguno de los 34 potenciales miembros contempla, de manera seria, inyectarle componentes adicionales de integración a este complicado proyecto.

\section{REFERENCIAS}

AlbA, Francisco (1993), «El Tratado de Libre Comercio y la emigración de mexicanos a Estados Unidos», en Comercio Exterior, vol. 43, núm. 8, agosto, pp. 743-749.

Alba, Francisco (1998), «Asimetrías, liberalización comercial y migración internacional: enseñanzas del caso europeo», en René M. Zenteno (coord.), Población, desarrollo y globalización, vol. 2, Sociedad Mexicana de Demografía, El Colegio de la Frontera Norte, México, pp. 17-30.

Ampi News (2003), «A turning point for EU migration policy», en George A. Papandreou, mayo 17-18. Disponible en «www.migrationpolicy.org/AMPI.news.html».

Andreas, Peter (2003), «A Tale of Two Borders: The U.S.-Canada and U.s. Mexico Lines after 9-11», en Peter Andreas y Thomas J. Biersteker (eds.), The Rebordering of North America. Integration and Exclusion in a New Security Context, Routledge, Nueva York, pp. 1-23.

BoerI, Tito y Herbert Brücker (2001), «Eastern Enlargement and Eu labor markets: Perceptions, challenges and opportunities», en IZA Discussion Paper, núm. 256, Bonn.

Boeri, Tito, Gordon Hanson y Barry McCormick (ed.) (2002), Immigration Policy and the welfare system. A report for the Fondazione Rodolfo Debenedetti in association with The William Davidson Institute, Oxford University Press.

BrochmanN, G. (1999), «Controlling Immigration in Europe», en Brochmann y Hammar (eds.), Mechanisms of Immigration Control. A comparative analysis of European regulation policies, Berg, pp. 297-334.

Clarkson, Stephen (2003), «The View from the Attic: Toward a Gated Continental Community?», en Peter Andreas y Thomas J. Biersteker (eds.), The Rebordering of North America. Integration and Exclusion in a New Security Context, Routledge, Nueva York.

Com (2001), «The relationship between internal security and complying with international protection obligations and instruments», Commission Working Document, Bruselas, 5 de diciembre.

Fertig, M. y C. Schmidt (2002), «Mobility within Europe: what do we (still not) know?», en IZA Discussion Paper, núm. 447, Bonn.

García y Griego, Manuel (1993), «La emigración mexicana y el Tratado de Libre Comercio en América del Norte: dos argumentos», en Gustavo Vega (coord.), Liberación económica y libre comercio en América del Norte, El Colegio de México, México.

Hristoulas, Athanasios (2003), «Trading Places: Canada, Mexico and North Ameri- 
can Security», en Peter Andreas y Thomas J. Biersteker (eds.), The Rebordering of North America. Integration and Exclusion in a New Security Context, Routledge, Nueva York, pp. 24-45.

Hufbauer, Gary Clyde y Gustavo Vega-Cánovas (2003), «Whither nafta: A Common Frontier?», en Peter Andreas y Thomas J. Biersteker (eds.), The Rebordering of North America. Integration and Exclusion in a New Security Context, Routledge, Nueva York, pp. 128-152.

JHA (Justice and Home Affairs) Council, Bruselas, 20 de septiembre 2001, Conclusión 29.

MAssey, Douglas S. (1998), «March of Folly. U.s. Immigration Policy Under NAFTA», en The American Prospect, núm. 37, marzo-abril, pp. 22-33.

MAssey, Douglas S. et al. (2002), Beyond Smoke and Mirrors: Mexican Immigration in the Era of Economic Integration, Russell Sage, Nueva York.

MonAR, J. (2000), «Justice and Home Affairs in a wider Europe: the dynamics of inclusion and exclusion», ESRC One Europe or Several? Programme Working Paper 07/00, Sussex European Institute.

Pastore, F. (2002), «Just another European dream? Why did the communitarization of immigration and asylum policies almost fail and how we should review it». Documento presentado en el seminario European Migration and Refugee Policy: new developments. Cicero Foundation, Roma, 15 de noviembre.

SHAw, Jo (2002), «Sovereignty at the boundaries of the politic», en ESRC One Europe or Several? Programme Working Paper 44/02, Sussex European Institute.

SPencer, C. (2001), «The EU as a security actor in the Mediterranean: problems and prospects», en Fred Tanner (ed.), The EU as a security actor in the Mediterranean, ЕTH, Zurich, pp.9-30.

TAssinopoulos, A. y Heinz Werner (1998), «Mobility and migration of labor in the EU», en Mobility and migration of labor in the European Union and their specific implications for young people, Cedefop Document, pp. 5-98.

Weintraub, Sidney et al. (1997), «Responses to Migration Issues». Documento preparado para el Estudio Binacional México-Estados Unidos sobre Migración, septiembre. 